\title{
Some properties of meromorphic solutions of higher order linear difference equations
}

\author{
B. Belaïdi, Y. Benkarouba
}

Abstract: In this paper, we investigate the growth of solutions of the linear difference equations

$$
\begin{gathered}
A_{k}(z) f\left(z+c_{k}\right)+A_{k-1}(z) f\left(z+c_{k-1}\right)+\cdots+A_{1}(z) f\left(z+c_{1}\right)+A_{0}(z) f(z)=0, \\
A_{k}(z) f\left(z+c_{k}\right)+A_{k-1}(z) f\left(z+c_{k-1}\right)+\cdots+A_{1}(z) f\left(z+c_{1}\right)+A_{0}(z) f(z)=F(z),
\end{gathered}
$$

where $A_{k}(z), \cdots, A_{0}(z), F(z)(\not \equiv 0)$ are entire functions and $c_{k}, \cdots, c_{1}$ are distinct non-zero complex numbers. We extend some precedent results due to Liu and Mao [15].

Keywords: Complex linear difference equation, meromorphic solution, iterated $p$-order, iterated $p$-type.

\section{Introduction and main results}

In this paper, we use the standard notations of Nevanlinna's value distribution theory (see [7], [11], [17]). Recently, study of properties of meromorphic solutions of complex difference equations have become a subject of great interest from the viewpoint of Nevanlinna theory, due to the apparent role of the existence of such solutions of finite order for the integrability of discrete difference equations (see, e.g., $[1,4,5,13,14,15,16,18,19]$ ). The key result here is the difference analogue of the lemma on the logarithmic derivative obtained by Halburd-Korhonen $[9,10]$ and Chiang-Feng [6], independently.

In the rest of the paper, the linear measure of a set $E \subset(0,+\infty)$ is defined as

$$
m(E)=\int_{0}^{+\infty} \chi_{E}(t) d t
$$

and the logarithmic measure of a set $F \subset(1,+\infty)$ is defined by

$$
\operatorname{lm}(F)=\int_{1}^{+\infty} \frac{\chi_{F}(t)}{t} d t
$$

Manuscript received May 7, 2019; accepted November 15, 2019.

Benharrat Belaïdi and Yamina Benkarouba are with the Department of Mathematics and Informatics, Laboratory of Pure and Applied Mathematics, University of Mostaganem (UMAB), Mostaganem, Algeria 
where $\chi_{H}(t)$ is the characteristic function of a set $H$. Moreover, the upper and the lower densities of a set $E \subset(0,+\infty)$ are defined respectively by

$$
\begin{aligned}
& \overline{\text { dens } E}=\limsup _{r \rightarrow+\infty} \frac{m(E \cap[0, r])}{r}, \\
& \underline{\text { dens } E}=\liminf _{r \rightarrow+\infty} \frac{m(E \cap[0, r])}{r},
\end{aligned}
$$

and the upper and the lower logarithmic densities of a set $F \subset(1,+\infty)$ are defined respectively by

$$
\begin{aligned}
& \overline{\log \text { dens }} F=\limsup _{r \rightarrow+\infty} \frac{\operatorname{lm}(F \cap[1, r])}{\log r}, \\
& \underline{\log \text { dens } F}=\liminf _{r \rightarrow+\infty} \frac{\operatorname{lm}(F \cap[1, r])}{\log r} .
\end{aligned}
$$

Proposition 1.1 [2] For all $H \subset[1,+\infty)$ the following statements hold :

(i) If $\operatorname{lm}(H)=\infty$, then $m(H)=\infty$;

(ii) If $\overline{\text { dens }} H>0$, then $m(H)=\infty$;

(iii) If $\overline{\log d e n s} H>0$, then $\operatorname{lm}(H)=\infty$.

In the following, we recall some fundamental definitions which are used later.

For all $r \in \mathbb{R}$, we define $\exp _{1} r:=e^{r}$ and $\exp _{p+1} r:=\exp \left(\exp _{p} r\right), p \in \mathbb{N}$. We also define for all $r$ sufficiently $\operatorname{large} \log _{1} r:=\log r$ and $\log _{p+1} r:=\log \left(\log _{p} r\right), p \in \mathbb{N}$. Moreover, we denote by $\exp _{0} r:=r, \log _{0} r:=r, \log _{-1} r:=\exp _{1} r$ and $\exp _{-1} r:=\log _{1} r$, see [12] .

Definition $1.1[12]$ Let $p \geq 1$ be an integer. Then, the iterated $p$-order $\rho_{p}(f)$ of a meromorphic function $f$ is defined by

$$
\rho_{p}(f)=\limsup _{r \rightarrow+\infty} \frac{\log _{p} T(r, f)}{\log r}
$$

where $T(r, f)$ is the characteristic function of Nevanlinna (see, $[7,11,16]$ ). For $p=1$, this notation is called order and hyper-order when $p=2$. The iterated $p$-order $\rho_{p}(f)$ of an entire function $f$ is defined by

$$
\rho_{p}(f)=\limsup _{r \rightarrow+\infty} \frac{\log _{p} T(r, f)}{\log r}=\limsup _{r \rightarrow+\infty} \frac{\log _{p+1} M(r, f)}{\log r},
$$

where $M(r, f)=\max _{|z|=r}|f(z)|$. 
Definition 1.2 [3] Let $f$ be a meromorphic function of iterated $p$-order $\left(0<\rho_{p}(f)<\infty\right)$, the iterated $p$-type $\tau_{p}(f)$ of $f$ is defined by

$$
\tau_{p}(f)=\limsup _{r \rightarrow+\infty} \frac{\log _{p-1} T(r, f)}{r^{\rho_{p}(f)}}(p \geq 1 \text { an integer }) .
$$

In recent paper [6], Chiang and Feng investigated meromorphic solutions of the linear difference equation

$$
A_{k}(z) f(z+k)+A_{k-1}(z) f(z+k-1)+\cdots+A_{1}(z) f(z+1)+A_{0}(z) f(z)=0,
$$

where $A_{k}(z), \cdots ; A_{0}(z)$ are entire functions and proved the following result.

Theorem $\mathbf{A}[6]$ Let $A_{0}(z), \cdots, A_{k}(z)$ be polynomials. If there exists an integer $l(0 \leq l \leq k)$ such that

$$
\operatorname{deg}\left(A_{l}\right)>\max _{0 \leq l \leq k, j \neq l}\left\{\operatorname{deg}\left(A_{j}\right)\right\}
$$

holds, then every meromorphic solution $f(\not \equiv 0)$ of equation (1.1) satisfies $\rho(f) \geq 1$, where $\operatorname{deg}\left(A_{l}\right)$ denotes the degree of the polynomial $A_{l}$.

Theorem $\mathbf{B}[6]$ Let $A_{0}(z), \cdots, A_{k}(z)$ be entire functions. If there exists an integer $l(0 \leq l \leq$ k) such that

$$
\rho\left(A_{l}\right)>\max _{0 \leq l \leq k, j \neq l}\left\{\rho\left(A_{j}\right)\right\}
$$

holds, then every meromorphic solution $f(\not \equiv 0)$ of equation (1.1) satisfies $\rho(f) \geq \rho\left(A_{l}\right)+1$.

Note that in Theorems A and B, equation (1.1) has only one dominating coefficient $A_{l}$. For the case when there is no dominating coefficient and all coefficients are polynomials in equation (1.1), Chen [4] obtained an improvement of Theorem A.

Theorem C [4] Let $A_{0}(z), \cdots, A_{k}(z)$ be polynomials such that

$$
\operatorname{deg}\left(A_{0}+\cdots+A_{k}\right)=\max _{0 \leq j \leq k}\left\{\operatorname{deg}\left(A_{j}\right)\right\} \geq 1 .
$$

Then every finite order meromorphic solution $f(\not \equiv 0)$ of equation (1.1) satisfies $\rho(f) \geq 1$.

For the case when there is more than one of coefficients which have the maximal order, Laine and Yang [13] obtained the following result. 
Theorem D [13] Let $A_{0}(z), \cdots, A_{k}(z)$ be entire functions of finite order such that among those having the maximal order

$$
\rho=\max _{0 \leq j \leq k}\left\{\rho\left(A_{j}\right)\right\}
$$

exactly one has its type strictly greater than the others. Then for every meromorphic solution $f(\not \equiv 0)$ of equation

$$
A_{k}(z) f\left(z+c_{k}\right)+A_{k-1}(z) f\left(z+c_{k-1}\right)+\cdots+A_{1}(z) f\left(z+c_{1}\right)+A_{0}(z) f(z)=0,
$$

where $c_{k}, \cdots, c_{1}$ are non-zero distinct complex numbers, we have

$$
\rho(f) \geq \rho+1
$$

In the present paper, we continue to study the growth of solutions of some linear difference equations, we improve and extend Theorem A, Theorem B, Theorem C and Theorem $\mathrm{D}$ by using the concept of the iterated $p$-order for equation (1.2). We obtain the following results.

Theorem 1.1 Let $H$ be a complex set satisfying $\overline{\log \text { dens }}\{r=|z|: z \in H\}>0$, and let $A_{0}(z), \cdots, A_{k}(z)$ be entire functions of iterated $p$-order satisfying $\max _{0 \leq j \leq k}\left\{\rho_{p}\left(A_{j}\right)\right\} \leq \rho$. If there exists an integer $l(0 \leq l \leq k)$ such that for some constants $0 \leq \beta<\alpha$ and $\delta$ $(0<\delta<\rho)$ sufficiently small, we have

$$
\begin{gathered}
\left|A_{l}(z)\right| \geq \exp _{p}\left\{\alpha r^{\rho-\delta}\right\}, \\
\left|A_{j}(z)\right| \leq \exp _{p}\left\{\beta r^{\rho-\delta}\right\}, \quad j=0, \cdots, k, j \neq l,
\end{gathered}
$$

as $z \rightarrow \infty$ for $z \in H$, then every meromorphic solution $f(\not \equiv 0)$ of equation (1.2) satisfies

$$
\left\{\begin{aligned}
\rho(f) \geq \rho\left(A_{l}\right)+1, & \text { for } p=1, \\
\rho_{p}(f) \geq \rho_{p}\left(A_{l}\right), & \text { for } p \geq 2 .
\end{aligned}\right.
$$

Remark 1.1 The Theorem 1.1 was obtained by Liu and Mao [15] when $p=1$ and for equation (1.1) with $H$ is a complex set satisfying $\overline{\operatorname{den} s}\{r=|z|: z \in H\}>0$.

Theorem 1.2 Let $H$ be a complex set satisfying $\overline{\log d e n s}\{r=|z|: z \in H\}>0$, and let $A_{0}(z), \cdots, A_{k}(z)$ be entire functions satisfying $\max _{0 \leq j \leq k}\left\{\rho_{p}\left(A_{j}\right)\right\} \leq \rho$. If there exists an integer $l(0 \leq l \leq k)$ such that for some constants $0 \leq \beta<\alpha$ and $\delta(0<\delta<\rho)$ sufficiently small, we have

$$
\begin{gathered}
T\left(r, A_{l}\right) \geq \exp _{p-1}\left\{\alpha r^{\rho-\delta}\right\}, \\
T\left(r, A_{j}\right) \leq \exp _{p-1}\left\{\beta r^{\rho-\delta}\right\}, \quad(j=0, \cdots, k, j \neq l),
\end{gathered}
$$


as $z \rightarrow \infty$ for $z \in H$. Then the following statements hold:

(i) If $p=1$ and $0 \leq k \beta<\alpha$, then every meromorphic solution $f \not \equiv 0$ of equation (1.2) satisfies $\rho(f) \geq \rho\left(A_{l}\right)+1$.

(ii) If $p \geq 2$ and $0 \leq \beta<\alpha$, then every meromorphic solution $f \not \equiv 0$ of equation (1.2) satisfies $\rho_{p}(f) \geq \rho_{p}\left(A_{l}\right)$.

In the following theorem, we will add a condition on the type. When there exists more than one coefficient having the order $\infty$ in equation (1.2), we obtain the following result. Note that in this case Theorem D is invalid for $p=1$.

Theorem 1.3 Let $A_{0}(z), \cdots, A_{k}(z)$ be entire functions, and let $p \geq 1$ be an integer. If there exists an integer $l(0 \leq l \leq k)$ such that

$$
\begin{gathered}
\max \left\{\rho_{p+1}\left(A_{j}\right): j=0, \cdots, k, j \neq l\right\} \leq \rho_{p+1}\left(A_{l}\right),\left(0<\rho_{p+1}\left(A_{l}\right)<\infty\right), \\
\max \left\{\tau_{p+1}\left(A_{j}\right): \rho_{p+1}\left(A_{j}\right)=\rho_{p+1}\left(A_{l}\right)\right\}<\tau_{p+1}\left(A_{l}\right),\left(0<\tau_{p+1}\left(A_{l}\right)<\infty\right),
\end{gathered}
$$

then every meromorphic solution $f(\not \equiv 0)$ of equation (1.2) satisfies

$$
\rho_{p}(f)=\infty \quad \text { and } \quad \rho_{p+1}(f) \geq \rho_{p+1}\left(A_{l}\right) .
$$

Next we consider the properties of meromorphic solutions of the non-homogeneous linear difference equation corresponding to (1.2)

$$
A_{k}(z) f\left(z+c_{k}\right)+\cdots+A_{1}(z) f\left(z+c_{1}\right)+A_{0}(z) f(z)=F(z)
$$

where where $A_{k}(z), \cdots, A_{0}(z), F(z)(\not \equiv 0)$ are entire functions and $c_{k}, \cdots, c_{1}$ are distinct nonzero complex numbers.

Theorem 1.4 Let $A_{j}(z)(j=0, \cdots, k)$ satisfy the hypothesis of Theorem 1.3, and let $F(z)$ be an entire function. Then

(i) If $\rho_{p+1}(F)<\rho_{p+1}\left(A_{l}\right)$ or $\rho_{p+1}(F)=\rho_{p+1}\left(A_{l}\right), \tau_{p+1}(F)<\tau_{p+1}\left(A_{l}\right)$, then every meromorphic solution $f(\not \equiv 0)$ of equation (1.7) satisfies

$$
\rho_{p}(f)=\infty \quad \text { and } \quad \rho_{p+1}(f) \geq \rho_{p+1}\left(A_{l}\right) .
$$

(ii) If $\rho_{p+1}(F)>\rho_{p+1}\left(A_{l}\right)$, then every meromorphic solution $f(\not \equiv 0)$ of equation (1.7) satisfies

$$
\rho_{p}(f)=\infty \quad \text { and } \quad \rho_{p+1}(f) \geq \rho_{p+1}(F) .
$$

Remark 1.2 The Theorems 1.3 and 1.4 were obtained by Liu and Mao [15] when $p=1$ and for equation (1.1). For some related results when $A_{j}(z)(j=0, \cdots, k), F(z)$ are meromorphic functions, see [19]. 


\section{Preliminary Lemmas}

Our proofs depend mainly upon the following lemmas.

Lemma 2.1 [6] Let $f$ be a meromorphic function, $\eta$ a non-zero complex number, and let $\gamma>1$, and $\varepsilon>0$ be given real constants. Then there exists a subset $E_{1} \subset(1,+\infty)$ of finite logarithmic measure, and a constant A depending only on $\gamma$ and $\eta$, such that for all $|z|=r \notin E_{1} \cup[0,1]$, we have

$$
|\log | \frac{f(z+\eta)}{f(z)}|| \leq A\left(\frac{T(\gamma r, f)}{r}+\frac{n(\gamma r)}{r} \log ^{\gamma} r \log ^{+} n(\gamma r)\right),
$$

where $n(t)=n(t, \infty, f)+n(t, \infty, 1 / f)$.

Lemma 2.2 [8] Let $f$ be a transcendental meromorphic function, let $j$ be non-negative integer, let $x$ be a value in the extended complex plane, and let $\mu>1$ be a real constant. Then there exists a constant $R>0$ such that for all $r>R$, we have

$$
n\left(r, x, f^{(j)}\right) \leq \frac{2 j+6}{\log \mu} T(\mu r, f) .
$$

Lemma 2.3 Let $f$ be a meromorphic function, $\eta$ a non-zero complex number, and $\varepsilon>0$ be given real constants. Then there exists a subset $E_{2} \subset(1,+\infty)$ of finite logarithmic measure, such that if $f$ has finite iterated $p$-order $\rho_{p}(f)=\rho$, then for all $|z|=r \notin[0,1] \cup E_{2}$, we have

(i) If $p=1$, then

$$
\exp \left\{-r^{\rho-1+\varepsilon}\right\} \leq\left|\frac{f(z+\eta)}{f(z)}\right| \leq \exp \left\{r^{\rho-1+\varepsilon}\right\}
$$

(ii) If $p \geq 2$, then

$$
\exp _{p}\left\{-r^{\rho+\varepsilon}\right\} \leq\left|\frac{f(z+\eta)}{f(z)}\right| \leq \exp _{p}\left\{r^{\rho+\varepsilon}\right\} .
$$

Proof. We prove only (ii). For the proof of (i) see [6]. Let $p \geq 2$. By Lemma 2.1, there exist a subset $E_{2} \subset(1,+\infty)$ of finite logarithmic measure, and a constant $A$ depending only on $\gamma$ and $\eta$, such that for all $|z|=r \notin E_{2} \cup[0,1]$, we have

$$
|\log | \frac{f(z+\eta)}{f(z)}|| \leq A\left(\frac{T(\gamma r, f)}{r}+\frac{n(\gamma r)}{r} \log ^{\gamma} r \log ^{+} n(\gamma r)\right),
$$

where $n(t)=n(t, \infty, f)+n(t, \infty, 1 / f)$. By using (2.1) and (2.4), we obtain

$$
|\log | \frac{f(z+\eta)}{f(z)}|| \leq A\left(\frac{T(\gamma r, f)}{r}\right.
$$




$$
\begin{gathered}
\left.+\frac{12}{\log \mu} \frac{T(\mu \gamma r, f)}{r} \log ^{\gamma} r \log ^{+}\left(\frac{12}{\log \mu} T(\mu \gamma r, f)\right)\right) \\
\leq B\left(T(\lambda r, f) \frac{\log ^{\lambda} r}{r} \log T(\lambda r, f)\right),
\end{gathered}
$$

where $B>0$ is some constant and $\lambda=\mu \gamma>1$. Since $f$ has finite iterated $p-\operatorname{order} \rho_{p}(f)=$ $\rho$, so given $\varepsilon, 0<\varepsilon<2$, we have for sufficiently large $r$

$$
T(r, f) \leq \exp _{p-1}\left\{r^{\rho+\frac{\varepsilon}{2}}\right\} .
$$

Then by using (2.5) and (2.6), we obtain

$$
\begin{gathered}
|\log | \frac{f(z+\eta)}{f(z)}|| \leq B\left(T(\lambda r, f) \frac{\log ^{\lambda} r}{r} \log T(\lambda r, f)\right) \\
\leq B \exp _{p-1}\left\{(\lambda r)^{\rho+\frac{\varepsilon}{2}}\right\} \frac{\log ^{\lambda} r}{r} \log \exp _{p-1}\left\{(\lambda r)^{\rho+\frac{\varepsilon}{2}}\right\} \\
=B \exp _{p-1}\left\{(\lambda r)^{\rho+\frac{\varepsilon}{2}}\right\} \frac{\log ^{\lambda} r}{r} \exp _{p-2}\left\{(\lambda r)^{\rho+\frac{\varepsilon}{2}}\right\} \leq \exp _{p-1}\left\{r^{\rho+\varepsilon}\right\} .
\end{gathered}
$$

From (2.7) we easily obtain (2.3).

Lemma 2.4 Let $\eta_{1}, \eta_{2}$ be two arbitrary complex numbers such that $\eta_{1} \neq \eta_{2}$, and let $f$ be a meromorphic function of finite iterated $p$-order $\rho_{p}(f)=\rho$. Let $\varepsilon>0$ be given, then there exists a subset $E_{3} \subset(0,+\infty)$ with finite logarithmic measure such that for all $|z|=r \notin E_{3} \cup[0,+\infty]$, we have

(i) If $p=1$, then

$$
\exp \left\{-r^{\rho-1+\varepsilon}\right\} \leq\left|\frac{f\left(z+\eta_{1}\right)}{f\left(z+\eta_{2}\right)}\right| \leq \exp \left\{r^{\rho-1+\varepsilon}\right\}
$$

(ii) If $p \geq 2$, then

$$
\exp _{p}\left\{-r^{\rho+\varepsilon}\right\} \leq\left|\frac{f\left(z+\eta_{1}\right)}{f\left(z+\eta_{2}\right)}\right| \leq \exp _{p}\left\{r^{\rho+\varepsilon}\right\}
$$

Proof. We prove only (ii). For the proof of (i) see [6]. We can write

$$
\left|\frac{f\left(z+\eta_{1}\right)}{f\left(z+\eta_{2}\right)}\right|=\left|\frac{f\left(z+\eta_{2}+\eta_{1}-\eta_{2}\right)}{f\left(z+\eta_{2}\right)}\right|, \quad\left(\eta_{1} \neq \eta_{2}\right) .
$$

Then by using Lemma 2.3, we obtain for any given $\varepsilon>0$ and all $\left|z+\eta_{2}\right|=R \notin[0,1] \cup E_{2}$, such that $\operatorname{lm}\left(E_{2}\right)<\infty$

$$
\exp _{p}\left\{-r^{\rho+\varepsilon}\right\} \leq \exp \left\{-\left(|z|+\left|\eta_{2}\right|\right)^{\rho+\frac{\varepsilon}{2}}\right\} \leq \exp _{p}\left\{-R^{\rho+\frac{\varepsilon}{2}}\right\}
$$




$$
\begin{gathered}
\leq\left|\frac{f\left(z+\eta_{1}\right)}{f\left(z+\eta_{2}\right)}\right|=\left|\frac{f\left(z+\eta_{2}+\eta_{1}-\eta_{2}\right)}{f\left(z+\eta_{2}\right)}\right| \\
\leq \exp _{p}\left\{R^{\rho+\frac{\varepsilon}{2}}\right\} \leq \exp _{p}\left\{\left(|z|+\left|\eta_{2}\right|\right)^{\rho+\frac{\varepsilon}{2}}\right\} \leq \exp _{p}\left\{r^{\rho+\varepsilon}\right\},
\end{gathered}
$$

where $|z|=r \notin[0,1] \cup E_{3}$ and $E_{3}$ is a set of finite logarithmic measure.

Lemma 2.5 [9] Let $f$ be a non-constant meromorphic function, $c \in \mathbb{C}, \delta<1$ and $\varepsilon>0$. Then

$$
m\left(r, \frac{f(z+c)}{f(z)}\right)=o\left(\frac{T(r+|c|, f)^{1+\varepsilon}}{r^{\delta}}\right)
$$

for all $r$ outside of a possible exceptional set $E_{4}$ with finite logarithmic measure $\int_{E_{4}} \frac{d r}{r}<\infty$.

Remark 2.1 [7] Let $f$ be a meromorphic function, c be a non-zero complex constant. Then we have that for $r \longrightarrow+\infty$

$$
(1+o(1)) T(r-|c|, f(z)) \leq T(r, f(z+c)) \leq(1+o(1)) T(r+|c|, f(z)) .
$$

Consecontly for $p \in \mathbb{N}_{+}=\{1,2, \cdots\}, \rho_{p}(f(z+h))=\rho_{p}(f)$.

Lemma 2.5 and Remark 2.1 lead to the following lemma.

Lemma 2.6 [9] Let $f$ be a non-constant meromorphic function, $c, h \in \mathbb{C}, c \neq h, \delta<1$, $\varepsilon>0$. Then

$$
m\left(r, \frac{f(z+c)}{f(z+h)}\right)=o\left(\frac{(T(r+|c-h|+|h|, f))^{1+\varepsilon}}{r^{\delta}}\right),
$$

holds for all $r$ outside of a possible exceptional set $E_{5}$ with finite logarithmic measure $\int_{E_{5}} \frac{d r}{r}<\infty$.

Lemma 2.7 [3] Let $f$ be a meromorphic function with iterated $p$-order $0<\rho_{p}(f)<\infty$ and iterated $p$-type $0<\tau_{p}(f)<\infty$. Then for any given $\beta<\tau_{p}(f)$, there exists a subset $E_{6} \subset[1,+\infty)$ of infinite logarithmic measure such that

$$
\log _{p-1} T(r, f)>\beta r^{\rho_{p}(f)},
$$

holds for all $r \in E_{6}$.

Lemma 2.8 [6] Let $\mu, R, R^{\prime}$ be real numbers such that $0<\mu<1, R>0$, and let $\eta$ be a non-zero complex number. Then there is a positive constant $C_{\mu}$ depending only on $\mu$ such 
that for a given meromorphic function $f$ we have, when $|z|=r, \max \{1, r+|\eta|\}<R<R^{\prime}$, the estimate

$$
\begin{gathered}
m\left(r, \frac{f(z+\eta)}{f(z)}\right)+m\left(r, \frac{f(z)}{f(z+\eta)}\right) \leq \frac{2|\eta| R}{(R-r-|\eta|)^{2}}\left(m(R, f)+m\left(R, \frac{1}{f}\right)\right) \\
+\frac{2 R^{\prime}}{\left(R^{\prime}-R\right)}\left(\frac{|\eta|}{R-r-|\eta|}+\frac{C_{\mu}|\eta|^{\mu}}{(1-\mu) r^{\mu}}\right)\left(N\left(R^{\prime}, f\right)+N\left(R^{\prime}, \frac{1}{f}\right)\right) .
\end{gathered}
$$

Lemma 2.9 Let $\eta_{1}, \eta_{2}$ be two complex numbers such that $\eta_{1} \neq \eta_{2}$ and let $f$ be a finite iterated $p$-order meromorphic function. Let $\rho_{p}(f)=\rho$ be the iterated $p$-order of $f$. Then for each $\varepsilon>0$, we have

(i) If $p=1$, then

$$
m\left(r, \frac{f\left(z+\eta_{1}\right)}{f\left(z+\eta_{2}\right)}\right)=O\left(r^{\rho-1+\varepsilon}\right)
$$

(ii) If $p \geq 2$, then

$$
m\left(r, \frac{f\left(z+\eta_{1}\right)}{f\left(z+\eta_{2}\right)}\right)=O\left(\exp _{p-1}\left\{r^{\rho+\varepsilon}\right\}\right) .
$$

Proof. We prove only (ii). For the proof of (i) see [6]. Let $p \geq 2$. We have

$$
\begin{gathered}
m\left(r, \frac{f\left(z+\eta_{1}\right)}{f\left(z+\eta_{2}\right)}\right) \leq m\left(r, \frac{f\left(z+\eta_{1}\right)}{f(z)}\right)+m\left(r, \frac{f(z)}{f\left(z+\eta_{2}\right)}\right) \\
\leq m\left(r, \frac{f\left(z+\eta_{1}\right)}{f(z)}\right)+m\left(r, \frac{f(z)}{f\left(z+\eta_{1}\right)}\right) \\
+m\left(r, \frac{f(z)}{f\left(z+\eta_{2}\right)}\right)+m\left(r, \frac{f\left(z+\eta_{2}\right)}{f(z)}\right) .
\end{gathered}
$$

Since $f$ has finite iterated $p$-order $\rho_{p}(f)=\rho<+\infty$, so given $\varepsilon, 0<\varepsilon<2$, we have

$$
T(r, f) \leq \exp _{p-1}\left\{r^{\rho+\frac{\varepsilon}{2}}\right\}
$$

for all $r$. By using Lemma 2.8, we obtain from equation (2.8)

$$
\begin{gathered}
m\left(r, \frac{f\left(z+\eta_{1}\right)}{f\left(z+\eta_{2}\right)}\right) \leq \frac{2\left|\eta_{1}\right| R}{\left(R-r-\left|\eta_{1}\right|\right)^{2}}\left(m(R, f)+m\left(R, \frac{1}{f}\right)\right) \\
+\frac{2 R^{\prime}}{\left(R^{\prime}-R\right)}\left(\frac{\left|\eta_{1}\right|}{R-r-\left|\eta_{1}\right|}+\frac{C_{\mu}\left|\eta_{1}\right|^{\mu}}{(1-\mu) r^{\mu}}\right)\left(N\left(R^{\prime}, f\right)+N\left(R^{\prime}, \frac{1}{f}\right)\right) \\
+\frac{2\left|\eta_{2}\right| R}{\left(R-r-\left|\eta_{2}\right|\right)^{2}}\left(m(R, f)+m\left(R, \frac{1}{f}\right)\right)
\end{gathered}
$$




$$
\begin{gathered}
+\frac{2 R^{\prime}}{\left(R^{\prime}-R\right)}\left(\frac{\left|\eta_{2}\right|}{R-r-\left|\eta_{2}\right|}+\frac{C_{\mu}\left|\eta_{2}\right|^{\mu}}{(1-\mu) r^{\mu}}\right)\left(N\left(R^{\prime}, f\right)+N\left(R^{\prime}, \frac{1}{f}\right)\right) \\
=\left(\frac{2\left|\eta_{1}\right| R}{\left(R-r-\left|\eta_{1}\right|\right)^{2}}+\frac{2\left|\eta_{2}\right| R}{\left(R-r-\left|\eta_{2}\right|\right)^{2}}\right)\left(m(R, f)+m\left(R, \frac{1}{f}\right)\right) \\
\quad+\frac{2 R^{\prime}}{\left(R^{\prime}-R\right)}\left(\frac{\left|\eta_{1}\right|}{R-r-\left|\eta_{1}\right|}+\frac{C_{\mu}\left|\eta_{1}\right|^{\mu}}{(1-\mu) r^{\mu}}\right. \\
\left.+\frac{\left|\eta_{2}\right|}{R-r-\left|\eta_{2}\right|}+\frac{C_{\mu}\left|\eta_{2}\right|^{\mu}}{(1-\mu) r^{\mu}}\right)\left(N\left(R^{\prime}, f\right)+N\left(R^{\prime}, \frac{1}{f}\right)\right) .
\end{gathered}
$$

By choosing $\mu=1-\frac{\varepsilon}{2}, R=2 r, R^{\prime}=3 r$ and $r>\max \left\{\left|\eta_{1}\right|,\left|\eta_{2}\right|, 1 / 2\right\}$ in (2.10), we get

$$
\begin{gathered}
m\left(r, \frac{f\left(z+\eta_{1}\right)}{f\left(z+\eta_{2}\right)}\right) \leq\left(\frac{4\left|\eta_{1}\right| r}{\left(r-\left|\eta_{1}\right|\right)^{2}}+\frac{4\left|\eta_{2}\right| r}{\left(r-\left|\eta_{2}\right|\right)^{2}}\right)\left(m(2 r, f)+m\left(2 r, \frac{1}{f}\right)\right) \\
+6\left(\frac{\left|\eta_{1}\right|}{r-\left|\eta_{1}\right|}+\frac{2 C_{\mu}\left|\eta_{1}\right|^{1-\frac{\varepsilon}{2}}}{\varepsilon r^{1-\frac{\varepsilon}{2}}}+\frac{\left|\eta_{2}\right|}{r-\left|\eta_{2}\right|}+\frac{2 C_{\mu}\left|\eta_{2}\right|^{1-\frac{\varepsilon}{2}}}{\varepsilon r^{1-\frac{\varepsilon}{2}}}\right)\left(N(3 r, f)+N\left(3 r, \frac{1}{f}\right)\right) \\
\leq 4\left[\frac{4\left|\eta_{1}\right| r}{\left(r-\left|\eta_{1}\right|\right)^{2}}+\frac{4\left|\eta_{2}\right| r}{\left(r-\left|\eta_{2}\right|\right)^{2}}\right. \\
\left.+6\left(\frac{\left|\eta_{1}\right|}{r-\left|\eta_{1}\right|}+\frac{\left|\eta_{2}\right|}{r-\left|\eta_{2}\right|}+\frac{2 C_{\mu}\left(\left|\eta_{1}\right|^{1-\frac{\varepsilon}{2}}+\left|\eta_{2}\right|^{1-\frac{\varepsilon}{2}}\right)}{\varepsilon r^{1-\frac{\varepsilon}{2}}}\right)\right] T(3 r, f) .
\end{gathered}
$$

From this, by using the estimate (2.9), we have

$$
\begin{gathered}
m\left(r, \frac{f\left(z+\eta_{1}\right)}{f\left(z+\eta_{2}\right)}\right) \leq 4 K\left[\frac{4\left|\eta_{1}\right| r}{\left(r-\left|\eta_{1}\right|\right)^{2}}+\frac{4\left|\eta_{2}\right| r}{\left(r-\left|\eta_{2}\right|\right)^{2}}\right. \\
\left.+6\left(\frac{\left|\eta_{1}\right|}{r-\left|\eta_{1}\right|}+\frac{\left|\eta_{2}\right|}{r-\left|\eta_{2}\right|}+\frac{2 C_{\mu}\left(\left|\eta_{1}\right|^{1-\frac{\varepsilon}{2}}+\left|\eta_{2}\right|^{1-\frac{\varepsilon}{2}}\right)}{\varepsilon r^{1-\frac{\varepsilon}{2}}}\right)\right] \exp _{p-1}\left\{(3 r)^{\rho+\frac{\varepsilon}{2}}\right\} \\
\leq M \exp _{p-1}\left\{r^{\rho+\varepsilon}\right\},
\end{gathered}
$$

where $K>0, M>0$ are some constants. This completes the proof.

Lemma 2.10 Under the assumptions of Theorem 1.1 or Theorem 1.2, we have $\rho_{p}\left(A_{l}\right)=\rho$.

Proof. By Theorem 1.1, we have $\rho_{p}\left(A_{l}\right) \leq \rho$. Suppose that $\rho_{p}\left(A_{l}\right)=\mu<\rho$. Then, for any given $\varepsilon>0$ and sufficiently large $r$, we have

$$
\left|A_{l}(z)\right| \leq \exp _{p}\left\{r^{\mu+\varepsilon}\right\} .
$$


On the other hand, by the hypotheses of Theorems 1.1, there exist positive constants $0 \leq$ $\beta<\alpha$ and $\delta(0<\delta<\rho)$ sufficiently small, such that

$$
\left|A_{l}(z)\right| \geq \exp _{p}\left\{\alpha r^{\rho-\delta}\right\}
$$

as $z \rightarrow \infty$ for $z \in H$. From (2.11) and (2.12), we obtain for $z \in H,|z|=r \rightarrow+\infty$

$$
\exp _{p}\left\{\alpha r^{\rho-\delta}\right\} \leq\left|A_{l}(z)\right| \leq \exp _{p}\left\{r^{\mu+\varepsilon}\right\}
$$

and by $\varepsilon$ is arbitrary with $0<\varepsilon<\rho-\mu-2 \delta$, this is a contradiction as $r \rightarrow+\infty$. Hence, $\rho_{p}\left(A_{l}\right)=\rho$.

By Theorem 1.2, we have $\rho_{p}\left(A_{l}\right) \leq \rho$. Suppose that $\rho_{p}\left(A_{l}\right)=\mu<\rho$. Then, for any given $\varepsilon>0$ and sufficiently large $r$, we have

$$
T\left(r, A_{l}\right) \leq \exp _{p-1}\left\{r^{\mu+\varepsilon}\right\} .
$$

On the other hand, by the hypotheses of Theorems 1.2, there exist positive constants $0 \leq$ $\beta<\alpha$ and $\delta(0<\delta<\rho)$ sufficiently small, such that

$$
T\left(r, A_{l}\right) \geq \exp _{p-1}\left\{\alpha r^{\rho-\delta}\right\}
$$

as $z \rightarrow \infty$ for $z \in H$. From (2.13) and (2.14), we obtain for $z \in H,|z|=r \rightarrow+\infty$

$$
\exp _{p-1}\left\{\alpha r^{\rho-\delta}\right\} \leq T\left(r, A_{l}\right) \leq \exp _{p-1}\left\{r^{\mu+\varepsilon}\right\}
$$

and by $\varepsilon$ is arbitrary with $0<\varepsilon<\rho-\mu-2 \delta$, this is a contradiction as $r \rightarrow+\infty$. Hence, $\rho_{p}\left(A_{l}\right)=\rho$.

\section{Proofs of main results}

Proof of Theorem 1.1. First case: When $p=1$, let $f(\not \equiv 0)$ be a meromorphic solution of equation (1.2). Suppose that $\rho(f)<\rho+1$. Then by Lemma 2.4 (i), for any given $\varepsilon(0<$ $\varepsilon<\rho+1-\rho(f)-2 \delta)$, there exists a set $E_{3} \subset(0,+\infty)$ with finite logarithmic measure such that for all $|z|=r \notin E_{3} \cup[0,1]$, we have

$$
\left|\frac{f\left(z+c_{j}\right)}{f\left(z+c_{l}\right)}\right| \leq \exp \left\{r^{\rho(f)-1+\varepsilon}\right\}<\exp \left\{r^{\rho-2 \delta}\right\}, \quad(j=1, \cdots, k, j \neq l)
$$

and

$$
\left|\frac{f(z)}{f\left(z+c_{l}\right)}\right| \leq \exp \left\{r^{\rho(f)-1+\varepsilon}\right\}<\exp \left\{r^{\rho-2 \delta}\right\} .
$$


We divide through equation $(1.2)$ by $f\left(z+c_{l}\right)$ to get

$$
\begin{gathered}
-A_{l}(z)=A_{k}(z) \frac{f\left(z+c_{k}\right)}{f\left(z+c_{l}\right)}+\cdots+A_{l-1}(z) \frac{f\left(z+c_{l-1}\right)}{f\left(z+c_{l}\right)} \\
+\cdots+A_{1}(z) \frac{f\left(z+c_{1}\right)}{f\left(z+c_{l}\right)}+A_{0}(z) \frac{f(z)}{f\left(z+c_{l}\right)} .
\end{gathered}
$$

Rewrite equation (3.3) in the form

$$
-1=\sum_{j=1, j \neq l}^{k} \frac{A_{j}(z) f\left(z+c_{j}\right)}{A_{l}(z) f\left(z+c_{l}\right)}+\frac{A_{0}(z) f(z)}{A_{l}(z) f\left(z+c_{l}\right)},
$$

it follows that

$$
1 \leq \sum_{j=1, j \neq l}^{k}\left|\frac{A_{j}(z)}{A_{l}(z)} \cdot \frac{f\left(z+c_{j}\right)}{f\left(z+c_{l}\right)}\right|+\left|\frac{A_{0}(z)}{A_{l}(z)} \cdot \frac{f(z)}{f\left(z+c_{l}\right)}\right| .
$$

From the conditions of Theorem 1.1, there is a set $H$ of complex numbers satisfying $\overline{\log \text { dens }}\{|z|: z \in H\}>0$ such that for $z \in H$, we have (1.3) and (1.4) as $|z| \rightarrow+\infty$. Set $H_{1}=\{r=|z|: z \in H\}$, since $\overline{\log d e n s}\{|z|: z \in H\}>0$, then $H_{1}$ is a set of $r$ with $\int_{H_{1}} \frac{d r}{r}=\infty$. Substituting (1.3), (1.4) (when $p=1$ ), (3.1) and (3.2) into (3.4), we get for $z \in H_{1} \backslash$ $\left(E_{3} \cup[0,1]\right)$

$$
1 \leq k \frac{\exp \left\{\beta r^{\rho-\delta}\right\}}{\exp \left\{\alpha r^{\rho-\delta}\right\}} \exp \left\{r^{\rho-2 \delta}\right\}=\exp \left\{(\beta-\alpha) r^{\rho-\delta}+r^{\rho-2 \delta}\right\} \rightarrow 0, r \rightarrow+\infty
$$

which is a contradiction. Hence, we get $\rho(f) \geq \rho+1$. By Lemma 2.10, we know that $\rho\left(A_{l}\right)=\rho$. So, $\rho(f) \geq \rho\left(A_{l}\right)+1$.

Second case: For $p \geq 2$, let $f(\not \equiv 0)$ be a meromorphic solution of equation (1.2). Suppose that $\rho_{p}(f)<\rho$. Then by Lemma 2.4 (ii), for any given $\varepsilon\left(0<\varepsilon<\rho-\rho_{p}(f)-2 \delta\right)$, there exists a set $E_{3} \subset(0,+\infty)$ with finite logarithmic measure such that for all $|z|=r \notin E_{3} \cup[0,1]$, we have

$$
\left|\frac{f\left(z+c_{j}\right)}{f\left(z+c_{l}\right)}\right| \leq \exp _{p}\left\{r^{\rho_{p}(f)+\varepsilon}\right\}<\exp _{p}\left\{r^{\rho-2 \delta}\right\}, \quad(j=1, \cdots, k, j \neq l)
$$

and

$$
\left|\frac{f(z)}{f\left(z+c_{l}\right)}\right| \leq \exp _{p}\left\{r^{\rho_{p}(f)+\varepsilon}\right\}<\exp _{p}\left\{r^{\rho-2 \delta}\right\}
$$

Substituting (1.3), (1.4), (3.5) and (3.6) into (3.4), we get for $z \in H_{1} \backslash\left(E_{3} \cup[0,1]\right)$

$$
1 \leq k \frac{\exp _{p}\left\{\beta r^{\rho-\delta}\right\}}{\exp _{p}\left\{\alpha r^{\rho-\delta}\right\}} \exp _{p}\left\{r^{\rho-2 \delta}\right\} \rightarrow 0, r \rightarrow+\infty
$$


which is a contradiction. Hence, we get $\rho_{p}(f) \geq \rho$. By Lemma 2.10, we know that $\rho_{p}\left(A_{l}\right)=$ $\rho$. So, $\rho_{p}(f) \geq \rho_{p}\left(A_{l}\right)$.

\section{Proof of Theorem 1.2}

First case: When $p=1$, let $f(\not \equiv 0)$ be a meromorphic solution of equation (1.2). Suppose that

$$
\rho(f)<\rho+1 .
$$

Since $A_{0}(z), \cdots, A_{k}(z)$ are entire functions, then by (3.3), we have

$$
\begin{gathered}
m\left(r, A_{l}(z)\right)=T\left(r, A_{l}(z)\right) \leq \sum_{j=0, j \neq l}^{k} m\left(r, A_{j}(z)\right) \\
+\sum_{j=1, j \neq l}^{k} m\left(r, \frac{f\left(z+c_{j}\right)}{f\left(z+c_{l}\right)}\right)+m\left(r, \frac{f(z)}{f\left(z+c_{l}\right)}\right)+O(1) \\
=\sum_{j=0, j \neq l}^{k} T\left(r, A_{j}(z)\right)+\sum_{j=1, j \neq l}^{k} m\left(r, \frac{f\left(z+c_{j}\right)}{f\left(z+c_{l}\right)}\right)+m\left(r, \frac{f(z)}{f\left(z+c_{l}\right)}\right)+O(1) .
\end{gathered}
$$

By Lemma 2.9 (i) and (3.7), we obtain for any given $\varepsilon(0<\varepsilon<\rho+1-\rho(f)-2 \delta)$

$$
\begin{gathered}
T\left(r, A_{l}(z)\right) \leq \sum_{j=0, j \neq l}^{k} T\left(r, A_{j}(z)\right)+\sum_{j=1, j \neq l}^{k} m\left(r, \frac{f\left(z+c_{j}\right)}{f\left(z+c_{l}\right)}\right) \\
+m\left(r, \frac{f(z)}{f\left(z+c_{l}\right)}\right)+O(1) \leq \sum_{j=0, j \neq l}^{k} T\left(r, A_{j}(z)\right)+O\left(r^{\rho(f)-1+\varepsilon}\right) .
\end{gathered}
$$

Substituting (1.5) and (1.6) (when $p=1$ ) into (3.8), we get for $|z|=r \rightarrow+\infty, z \in H$

$$
(\alpha-k \beta) r^{\rho-\delta} \leq O\left(r^{\rho(f)-1+\varepsilon}\right) .
$$

By $\alpha-k \beta>0$, it follows that

$$
1 \leq O(1) r^{\rho(f)-1+\varepsilon-\rho+\delta} \rightarrow 0, r \rightarrow+\infty
$$

which is a contradiction. Hence we get $\rho(f) \geq \rho+1$. By Lemma 2.10, we know that $\rho\left(A_{l}\right)=\rho$. So, $\rho(f) \geq \rho\left(A_{l}\right)+1$.

Second case: For $p \geq 2$, let $f(\not \equiv 0)$ be a meromorphic solution of equation (1.2). Suppose that $\rho_{p}(f)<\rho$. By Lemma 2.9 (ii) and (3.7), we obtain for any given $\varepsilon(0<\varepsilon<\rho-$ $\left.\rho_{p}(f)-2 \delta\right)$

$$
T\left(r, A_{l}(z)\right) \leq \sum_{j=0, j \neq l}^{k} T\left(r, A_{j}(z)\right)
$$




$$
+\sum_{j=1, j \neq l}^{k} \exp _{p-1}\left\{r^{\rho_{p}(f)+\varepsilon}\right\}+\exp _{p-1}\left\{r^{\rho_{p}(f)+\varepsilon}\right\}+O(1) .
$$

Substituting (1.5) and (1.6) (when $p=2$ ) into (3.9), we get for $|z|=r \rightarrow+\infty, z \in H$

$$
\begin{array}{r}
\exp _{p-1}\left\{\alpha r^{\rho-\delta}\right\} \leq \sum_{j=0, j \neq l}^{k} \exp _{p-1}\left\{\beta r^{\rho-\delta}\right\}+\sum_{j=1, j \neq l}^{k} \exp _{p-1}\left\{r^{\rho_{p}(f)+\varepsilon}\right\} \\
+\exp _{p-1}\left\{r^{\rho_{p}(f)+\varepsilon}\right\}+O(1) \leq k \exp _{p-1}\left\{\beta r^{\rho-\delta}\right\}+k \exp _{p-1}\left\{r^{\rho_{p}(f)+\varepsilon}\right\}+O(1) .
\end{array}
$$

By (3.10), we obtain

$$
(\alpha-\beta) r^{\rho-\delta} \leq r^{\rho_{p}(f)+\varepsilon}+O(1) .
$$

By $\alpha-\beta>0$, it follows that

$$
1 \leq \frac{1}{\alpha-\beta} r^{\rho_{p}(f)+\varepsilon-\rho+\delta}+\frac{1}{(\alpha-\beta) r^{\rho-\delta}} O(1) \rightarrow 0, r \rightarrow+\infty
$$

which is a contradiction. By Lemma 2.10, we know that $\rho_{p}\left(A_{l}\right)=\rho$. Hence, we get $\rho_{p}(f) \geq \rho_{p}\left(A_{l}\right)$. Thus, Theorem 1.2 is proved.

Proof of Theorem 1.3. Let $f(\not \equiv 0)$ be a meromorphic solution of (1.2). By equation (3.7) and Lemma 2.6, we obtain

$$
\begin{gathered}
T\left(r, A_{l}(z)\right)=m\left(r, A_{l}(z)\right) \leq \sum_{j=0, j \neq l}^{k} m\left(r, A_{j}(z)\right)+\sum_{j=1, j \neq l}^{k} m\left(r, \frac{f\left(z+c_{j}\right)}{f\left(z+c_{l}\right)}\right) \\
+m\left(r, \frac{f(z)}{f\left(z+c_{l}\right)}\right)+O(1) \leq \sum_{j=0, j \neq l}^{k} T\left(r, A_{j}(z)\right) \\
+\sum_{j=1, j \neq l}^{k} o\left(\frac{\left(T\left(r+\left|c_{j}-c_{l}\right|+\left|c_{l}\right|, f\right)\right)^{1+\varepsilon}}{r^{\delta}}\right) \\
+o\left(\frac{\left(T\left(r+2\left|c_{l}\right|, f\right)\right)^{1+\varepsilon}}{r^{\delta}}\right)+O(1) \leq \sum_{j=0, j \neq l}^{k} T\left(r, A_{j}(z)\right)+o\left(\frac{\left(T\left(r+2\left|c_{l}\right|, f\right)\right)^{1+\varepsilon}}{r^{\delta}}\right)
\end{gathered}
$$

for all $r$ outside of a possible exceptional set $E_{5}$ with finite logarithmic measure $\int_{E_{5}} \frac{d r}{r}<\infty$. Let $\beta_{1}, \beta_{2}$ be two real numbers such that

$$
\max \left\{\tau_{p+1}\left(A_{j}\right): \rho_{p+1}\left(A_{j}\right)=\rho_{p+1}\left(A_{l}\right)\right\}<\beta_{1}<\beta_{2}<\tau_{p+1}\left(A_{l}\right) .
$$


Then by Lemma 2.7, we know that there exists a set $E_{6}$ of infinite logarithmic measure, such that

$$
T\left(r, A_{l}\right)>\exp _{p}\left\{\beta_{2} r^{\rho_{p+1}\left(A_{l}\right)}\right\}
$$

holds for all $r \in E_{6}$. Therefore we can take a sequence $\left\{r_{n}\right\}$ such that $r_{n} \in E_{6}, r_{n} \rightarrow \infty$, and

$$
T\left(r_{n}, A_{l}\right)>\exp _{p}\left\{\beta_{2} r_{n}^{\rho_{p+1}\left(A_{l}\right)}\right\} .
$$

On the other hand, if $b=\max \left\{\rho_{p+1}\left(A_{j}\right): j=0, \cdots, k, j \neq l\right\}<\rho_{p+1}\left(A_{l}\right)$, then for any given $\varepsilon\left(0<\varepsilon<\rho_{p+1}\left(A_{l}\right)-b\right)$ and sufficiently large $r_{n}$, we have

$$
T\left(r_{n}, A_{j}\right) \leq \exp _{p}\left\{r_{n}^{b+\varepsilon}\right\} \leq \exp _{p}\left\{\beta_{1} r_{n}^{\rho_{p+1}\left(A_{l}\right)}\right\} .
$$

If $\max \left\{\tau_{p+1}\left(A_{j}\right): \rho_{p+1}\left(A_{j}\right)=\rho_{p+1}\left(A_{l}\right)\right\}<\tau_{p+1}\left(A_{l}\right)$, then for sufficiently large $r_{n}$, we have

$$
T\left(r_{n}, A_{j}\right) \leq \exp _{p}\left\{\beta_{1} r_{n}^{\rho_{p+1}\left(A_{l}\right)}\right\} .
$$

Then substituting (3.12), (3.13) or (3.14) into (3.11), we get for $r_{n} \in E_{6} \backslash E_{5}$

$$
\exp _{p}\left\{\beta_{2} r_{n}^{\rho_{p+1}\left(A_{l}\right)}\right\}<T\left(r_{n}, A_{l}\right) \leq k \exp _{p}\left\{\beta_{1} r_{n}^{\rho_{p+1}\left(A_{l}\right)}\right\}+o\left(\frac{\left(T\left(r_{n}+2\left|c_{l}\right|, f\right)\right)^{1+\varepsilon}}{r_{n}^{\delta}}\right) \text {. }
$$

Then by (3.15), we get

$$
(1-o(1)) \exp _{p}\left\{\beta_{2} r_{n}^{\rho_{p+1}\left(A_{l}\right)}\right\}<o\left(\frac{\left(T\left(r_{n}+2\left|c_{l}\right|, f\right)\right)^{1+\varepsilon}}{r_{n}^{\delta}}\right) .
$$

Hence,

$$
\rho_{p}(f)=\infty \quad \text { and } \quad \rho_{p+1}(f) \geq \rho_{p+1}\left(A_{l}\right) .
$$

Proof of Theorem 1.4. (i) First we consider the case

$$
\rho_{p+1}(F)<\rho_{p+1}\left(A_{l}\right) \quad \text { or } \quad \rho_{p+1}(F)=\rho_{p+1}\left(A_{l}\right), \tau_{p+1}(F)<\tau_{p+1}\left(A_{l}\right) .
$$

Let $f$ be a meromorphic solution of (1.7). We divide equation (1.7) by $f\left(z+c_{l}\right)$ to get

$$
-A_{l}(z)=\sum_{j=1, j \neq l}^{k} A_{j}(z) \frac{f\left(z+c_{j}\right)}{f\left(z+c_{l}\right)}+A_{0}(z) \frac{f(z)}{f\left(z+c_{l}\right)}-\frac{F(z)}{f\left(z+c_{l}\right)} .
$$

It follows from (3.16), Remark 2.1 and Lemma 2.6 that for any given $\varepsilon>0$ and sufficiently large $r$, we have

$$
T\left(r, A_{l}(z)\right)=m\left(r, A_{l}(z)\right) \leq m\left(r, \frac{F(z)}{f\left(z+c_{l}\right)}\right)+\sum_{j=0, j \neq l}^{k} m\left(r, A_{j}(z)\right)
$$




$$
\begin{array}{r}
+\sum_{j=1, j \neq l}^{k} m\left(r, \frac{f\left(z+c_{j}\right)}{f\left(z+c_{l}\right)}\right)+m\left(r, \frac{f(z)}{f\left(z+c_{l}\right)}\right)+O(1) \leq T(r, F(z)) \\
+T\left(r, f\left(z+c_{l}\right)\right)+\sum_{j=0, j \neq l}^{k} m\left(r, A_{j}(z)\right)+o\left(\frac{\left(T\left(r+2\left|c_{l}\right|, f\right)\right)^{1+\varepsilon}}{r^{\delta}}\right) \\
\leq T(r, F(z))+(1+o(1)) T\left(r+\left|c_{l}\right|, f(z)\right)+\sum_{j=0, j \neq l}^{k} T\left(r, A_{j}(z)\right) \\
+o\left(\frac{\left(T\left(r+2\left|c_{l}\right|, f\right)\right)^{1+\varepsilon}}{r^{\delta}}\right) \leq T(r, F(z)) \\
+\sum_{j=0, j \neq l}^{k} T\left(r, A_{j}(z)\right)+2 T\left(r+\left|c_{l}\right|, f(z)\right)+o\left(\frac{\left(T\left(r+2\left|c_{l}\right|, f\right)\right)^{1+\varepsilon}}{r^{\delta}}\right)
\end{array}
$$

for $r \rightarrow \infty, r \notin E_{5}$, where $E_{5}$ is a set of finite logarithmic measure. Let $\beta_{1}, \beta_{2}$ be two real numbers such that

$$
\max \left\{\tau_{p+1}\left(A_{j}\right), \tau_{p+1}(F): \rho_{p+1}\left(A_{j}\right)=\rho_{p+1}\left(A_{l}\right)\right\}<\beta_{1}<\beta_{2}<\tau_{p+1}\left(A_{l}\right) .
$$

Then by Lemma 2.7, we can take a sequence $\left\{r_{n}\right\}$ such that $r_{n} \in E_{6}, r_{n} \rightarrow \infty$, and equations (3.12)-(3.14) also hold for sufficiently large $r_{n}$. On the other hand, for sufficiently large $r_{n}$ we have

$$
T\left(r_{n}, F\right) \leq \exp _{p}\left\{\beta_{1} r_{n}^{\rho_{p+1}\left(A_{l}\right)}\right\} .
$$

Substituting equations (3.12), (3.13) (or (3.14)) and (3.18) into (3.17), we get for $r_{n} \in$ $E_{6} \backslash E_{5}$

$$
\exp _{p}\left\{\beta_{2} r_{n}^{\rho_{p+1}\left(A_{l}\right)}\right\}<T\left(r_{n}, A_{l}\right) \leq(k+1) \exp _{p}\left\{\beta_{1} r_{n}^{\rho_{p+1}\left(A_{l}\right)}\right\}+3\left(T\left(2 r_{n}, f\right)\right)^{2} .
$$

Hence, by equation (3.19), we get

$$
\rho_{p}(f)=\infty \quad \text { and } \quad \rho_{p+1}(f) \geq \rho_{p+1}\left(A_{l}\right) .
$$

(ii) Next we consider the case $\rho_{p+1}(F)>\rho_{p+1}\left(A_{l}\right)$. Let $f$ be a meromorphic solution of (1.7). By Remark 2.1 and Lemma 2.6 that for any given $\varepsilon>0$ and sufficiently large $r$, we have

$$
\begin{aligned}
& T(r, F(z)) \leq \sum_{j=0}^{k} T\left(r, A_{j}(z)\right)+\sum_{j=1}^{k} T\left(r, f\left(z+c_{j}\right)\right)+T(r, f(z))+O(1) \\
& \leq \sum_{j=0}^{k} T\left(r, A_{j}(z)\right)+(1+o(1)) k T\left(r, f\left(z+\left|c_{s}\right|\right)\right)+T(r, f(z))+O(1)
\end{aligned}
$$




$$
\leq \sum_{j=0}^{k} T\left(r, A_{j}(z)\right)+(2 k+1) T(2 r, f(z))+O(1),\left|c_{s}\right|=\max _{1 \leq j \leq k}\left\{\left|c_{j}\right|\right\} .
$$

By the definition of iterated $p+1$-order, we know that there exists a sequence $\left\{r_{n}\right\}$ such that $r_{n} \rightarrow+\infty$, and for any given $\varepsilon\left(0<2 \varepsilon<\rho_{p+1}(F)-\rho_{p+1}\left(A_{l}\right)\right)$, we have

$$
T\left(r_{n}, F\right) \geq \exp _{p}\left\{r_{n}^{\rho_{p+1}(F)-\varepsilon}\right\}
$$

and

$$
T\left(r_{n}, A_{j}\right) \leq \exp _{p}\left\{r_{n}^{b+\varepsilon}\right\} \leq \exp _{p}\left\{r_{n}^{\rho_{p+1}\left(A_{l}\right)+\varepsilon}\right\}(j=0, \cdots, k),
$$

where $b=\max \left\{\rho_{p+1}\left(A_{j}\right): j=0, \cdots, k, j \neq l\right\}<\rho_{p+1}\left(A_{l}\right)$. Substituting (3.21) and (3.22) into (3.20), we get

$$
\exp _{p}\left\{r_{n}^{\rho_{p+1}(F)-\varepsilon}\right\} \leq(k+1) \exp _{p}\left\{r_{n}^{\rho_{p+1}\left(A_{l}\right)+\varepsilon}\right\}+(2 k+1) T(2 r, f(z)) .
$$

Hence,

$$
\rho_{p}(f)=\infty \quad \text { and } \quad \rho_{p+1}(f) \geq \rho_{p+1}(F) .
$$

\section{Examples}

Next we give an example that illustrates Theorem 1.1.

Exemple 4.1. We consider the meromorphic function

$$
f(z)=e^{-z^{2}} \tan z
$$

Then $f$ satisfies the difference equation

$$
A_{2}(z) f(z+2 \pi)+A_{1}(z) f(z+\pi)+A_{0}(z) f(z)=0,
$$

where

$$
A_{2}(z)=2 \exp \left\{2 \pi z+3 \pi^{2}\right\}, A_{1}(z)=-1, A_{0}(z)=-\exp \left\{-2 \pi z-\pi^{2}\right\}
$$

We have

$$
\rho\left(A_{2}\right)=\rho\left(A_{0}\right)=1, \quad \rho\left(A_{1}\right)=0
$$

and

$$
1=\max _{0 \leq j \leq 1}\left\{\rho\left(A_{j}\right)\right\} \leq \rho=1 .
$$

We choose

$$
H=\left\{z \in \mathbb{C}: z=r e^{i \theta}, r \in\left[1,+\infty\left[, \frac{\pi}{4} \leq \theta \leq \frac{\pi}{3}\right\}\right.\right.
$$


a complex set satisfying $\overline{\log d e n s}\{r=|z|: z \in H\}>0$, we get for $\delta(0<\delta<\rho=1)$ sufficiently small

$$
\begin{gathered}
\left|A_{2}(z)\right|=\left|2 \exp \left\{2 \pi z+3 \pi^{2}\right\}\right|=2 \exp \left\{2 \pi r \cos \theta+3 \pi^{2}\right\} \\
\geq 2 \exp \left\{\pi r+4 \pi^{2}\right\} \geq \exp \left\{\pi r^{1-\delta}\right\}, \\
\left|A_{1}(z)\right|=1 \leq \exp \left\{r^{1-\delta}\right\}
\end{gathered}
$$

and

$$
\begin{gathered}
\left|A_{0}(z)\right|=\left|-\exp \left\{-2 \pi z-\pi^{2}\right\}\right|=\exp \left\{-2 \pi r \cos \theta-\pi^{2}\right\} \\
\leq \exp \left\{-\pi r-\pi^{2}\right\} \leq \exp \left\{r^{1-\delta}\right\}
\end{gathered}
$$

as $z \rightarrow \infty$ for $z \in H$. As we see, conditions of Theorem 1.1 are verified with $\alpha=\pi$ and $\beta=1$. We get

$$
2=\rho(f) \geq \rho\left(A_{2}\right)+1=2 .
$$

Next we give an example that illustrates Theorem 1.3.

Exemple 4.2. Consider the difference equation

$$
\begin{gathered}
(z+2 i \pi) \exp \{-\sin (z+2 i \pi)\} f(z+2 i \pi) \\
-2\left(z+i \frac{\pi}{2}\right) \exp \left\{2 i \sinh 2 z-\sin \left(z+i \frac{\pi}{2}\right)\right\} f\left(z+i \frac{\pi}{2}\right) \\
+z \exp \{-\sin z\} f(z)=0 .
\end{gathered}
$$

In this equation we have

$$
\begin{gathered}
A_{2}(z)=(z+2 i \pi) \exp \{-\sin (z+2 i \pi)\}, \\
A_{1}(z)=-2\left(z+i \frac{\pi}{2}\right) \exp \left\{2 i \sinh 2 z-\sin \left(z+i \frac{\pi}{2}\right)\right\}, \\
A_{0}(z)=z \exp \{-\sin z\} .
\end{gathered}
$$

We obtain

$$
\begin{gathered}
\rho\left(A_{2}\right)=\rho\left(A_{1}\right)=\rho\left(A_{0}\right)=\infty, \\
\rho_{2}\left(A_{2}\right)=\rho_{2}\left(A_{1}\right)=\rho_{2}\left(A_{0}\right)=1, \\
\tau_{2}\left(A_{2}\right)=1, \quad \tau_{2}\left(A_{1}\right)=2, \quad \tau_{2}\left(A_{0}\right)=1 .
\end{gathered}
$$

As we see, conditions of Theorem 1.3 are verified

$$
\begin{gathered}
1=\max \left\{\rho_{2}\left(A_{j}\right): j=0,2\right\} \leq \rho_{2}\left(A_{1}\right)=1, \\
1=\max \left\{\tau_{2}\left(A_{j}\right): \rho_{2}\left(A_{j}\right)=\rho_{2}\left(A_{1}\right)\right\}<\tau_{2}\left(A_{1}\right)=2 .
\end{gathered}
$$


The meromorphic function

$$
f(z)=\frac{\exp \{\sin 2 i z+\sin z\}}{z}
$$

is solution of equation (4.2) and $f$ satisfies

$$
\rho(f)=\infty \quad \text { and } \quad 1=\rho_{2}(f) \geq \rho_{2}\left(A_{1}\right)=1 .
$$

Next, we give an example that illustrates Theorem 1.4.

Exemple 4.3. Case (i). Consider the difference equation

$$
\begin{aligned}
& (z+2 i \pi) \exp \{-\sin (z+2 i \pi)\} f(z+2 i \pi) \\
& -(z+i \pi) \exp \{-\sin (z+i \pi)\} f(z+i \pi) \\
& +z \exp \{-\sin 2 i z\} f(z)=\exp \{\sin z\} .
\end{aligned}
$$

In this equation, we have

$$
\begin{gathered}
A_{2}(z)=(z+2 i \pi) \exp \{-\sin (z+2 i \pi)\}, \\
A_{1}(z)=-(z+i \pi) \exp \{-\sin (z+i \pi)\}, \\
A_{0}(z)=z \exp \{-\sin 2 i z\}, F(z)=\exp \{\sin z\} .
\end{gathered}
$$

We obtain

$$
\begin{gathered}
\rho\left(A_{2}\right)=\rho\left(A_{1}\right)=\rho\left(A_{0}\right)=\rho(F)=\infty, \\
\rho_{2}\left(A_{2}\right)=\rho_{2}\left(A_{1}\right)=\rho_{2}\left(A_{0}\right)=\rho_{2}(F)=1, \\
\tau_{2}\left(A_{2}\right)=1, \quad \tau_{2}\left(A_{1}\right)=1, \quad \tau_{2}\left(A_{0}\right)=2, \quad \tau_{2}(F)=1 .
\end{gathered}
$$

It clear that the conditions of Theorem 1.4 (i) are satisfied

$$
\begin{gathered}
1=\max \left\{\rho_{2}\left(A_{j}\right): j=1,2\right\} \leq \rho_{2}\left(A_{0}\right)=1, \\
1=\max \left\{\tau_{2}\left(A_{j}\right): \rho_{2}\left(A_{j}\right)=\rho_{2}\left(A_{0}\right)\right\}<\tau_{2}\left(A_{0}\right)=2, \\
\rho_{2}(F)=\rho_{2}\left(A_{0}\right)=1 \text { and } \tau_{2}(F)=1<\tau_{2}\left(A_{0}\right)=2 \text {. The meromorphic function } \\
f(z)=\frac{\exp \{\sin 2 i z+\sin z\}}{z}
\end{gathered}
$$

is solution of equation (4.3) and $f$ satisfies

$$
\rho(f)=\infty \quad \text { and } \quad 1=\rho_{2}(f) \geq \rho_{2}\left(A_{0}\right)=1 .
$$


Case (ii). We consider the meromorphic function

$$
f(z)=\frac{\exp \{\sin z\}}{z} .
$$

Then $f$ satisfies the difference equation

$$
A_{2}(z) f(z+2 \pi)+A_{1}(z) f(z+\pi)+A_{0}(z) f(z)=F(z),
$$

where

$$
\begin{gathered}
A_{2}(z)=(z+2 \pi) \exp \left\{\frac{\sin \sqrt{z}}{\sqrt{z}}\right\}, A_{1}(z)=(z+\pi) \exp \left\{\frac{\sin 3 \sqrt{z}}{\sqrt{z}}\right\} \\
A_{0}(z)=-z \exp \left\{\frac{\sin \sqrt{z}}{\sqrt{z}}\right\}, F(z)=\exp \left\{-\sin z+\frac{\sin 3 \sqrt{z}}{\sqrt{z}}\right\} .
\end{gathered}
$$

We have

$$
\begin{gathered}
\rho\left(A_{2}\right)=\rho\left(A_{1}\right)=\rho\left(A_{0}\right)=\rho(F)=\infty, \\
\rho_{2}(F)=1, \quad \rho_{2}\left(A_{2}\right)=\rho_{2}\left(A_{1}\right)=\rho_{2}\left(A_{0}\right)=1 / 2, \\
\tau_{2}\left(A_{2}\right)=\tau_{2}\left(A_{0}\right)=1, \quad \tau_{2}\left(A_{1}\right)=3 .
\end{gathered}
$$

It clear that the conditions of Theorem 1.4 (ii) are satisfied

$$
\begin{gathered}
1 / 2=\max \left\{\rho_{2}\left(A_{j}\right): j=0,2\right\} \leq \rho_{2}\left(A_{1}\right)=1 / 2, \\
1=\max \left\{\tau_{2}\left(A_{j}\right): \rho_{2}\left(A_{j}\right)=\rho_{2}\left(A_{1}\right)\right\}<3
\end{gathered}
$$

and $1=\rho_{2}(F)>\rho_{2}\left(A_{1}\right)=1 / 2$. We see that

$$
\rho(f)=\infty \quad \text { and } \quad 1=\rho_{2}(f) \geq \rho_{2}(F)=1 .
$$

Acknowledgements. The authors would like to thank the anonymous referee and editor for their helpful remarks and suggestions to improve this article. This paper is supported by University of Mostaganem (UMAB) (PRFU Project Code C00L03UN270120180005).

\section{References}

[1] M. J. Ablowitz, R. Halburd, B. Herbst, On the extension of the Painleve property to difference equations. Nonlinearity 13 (3) (2000) 889-905.

[2] B. BELAÏDI, On the [p,q]-order of meromorphic solutions of linear differential equations. Acta Univ. M. Belii Ser. Math. 23 (2015), 57-69.

[3] T. B. CAO, J. F. XU, Z. X. ChEN, On the meromorphic solutions of linear differential equations on the complex plane. J. Math. Anal. Appl. 364 (1) (2010) 130-142. 
[4] Z. X. CHEN, Growth and zeros of meromorphic solution of some linear difference equations. J. Math. Anal. Appl. 373 (1) (2011) 235-241.

[5] Z. X. CHEN, K. H. SHON, On growth of meromorphic solutions for linear difference equations. Abstr. Appl. Anal. 2013, Art. ID 619296, 1-6.

[6] Y. M. Chiang, S. J. Feng, On the Nevanlinna characteristic of $f(z+\eta)$ and difference equations in the complex plane, Ramanujan J. 16 (1)(2008) 105-129.

[7] A. Goldberg, I. OstrovskiI, Value Distribution of Meromorphic functions, Transl. Math. Monogr., vol. 236, Amer. Math. Soc., Providence RI, 2008.

[8] G. G. GUNDERSEN, Estimates for the logarithmic derivative of a meromorphic function, plus similar estimates. J. London Math. Soc. (2) 37 (1988) 88-104.

[9] R. G. Halburd, R. J. Korhonen, Difference analogue of the lemma on the logarithmic derivative with applications to difference equations, J. Math. Anal. Appl. 314 (2) (2006) $477-$ 487.

[10] R. G. Halburd, R. J. Korhonen, Nevanlinna theory for the difference operator. Ann. Acad. Sci. Fenn. Math. 31 (2) (2006) 463-478.

[11] W. K. Hayman, Meromorphic functions. Oxford Mathematical Monographs Clarendon Press, Oxford 1964.

[12] L. KINNUNEn, Linear differential equations with solutions of finite iterated order. Southeast Asian Bull. Math. 22 (4) (1998) 385-405.

[13] I. LAINE, C. C. YANG, Clunie theorems for difference and q-difference polynomials. J. Lond. Math. Soc. (2) 76 (2007) 556-566.

[14] Z. LATREUCH, B. BELAÏDI, Growth and oscillation of meromorphic solutions of linear difference equations. Mat. Vesnik 66 (2) (2014) 2, 213-222.

[15] H. F. LiU, Z. Q. MAO, On the meromorphic solutions of some linear difference equations. Adv. Difference Equ. 2013, 2013:133, 1-12.

[16] J. WANG, Growth and poles of meromorphic solutions of some difference equations. J. Math. Anal. Appl. 379(1) (2011) 367-377.

[17] C. C. YAng, H. X. YI, Uniqueness theory of meromorphic functions, Mathematics and its Applications, 557. Kluwer Academic Publishers Group, Dordrecht, 2003.

[18] X. M. ZHENG, J. TU, Growth of meromorphic solutions of linear difference equations. J. Math. Anal. Appl. 384 (2)(2011) 349-356.

[19] Y. P. ZHOU, X. M. ZHENG, Growth of meromorphic solutions to homogeneous and nonhomogeneous linear (differential-)difference equations with meromorphic coefficients. Electron. J. Differential Equations 2017, Paper No. 34, 15 pp. 